\title{
Explicit Runge-Kutta method with trigonometrically-fitted for solving first order ODEs
}

\begin{abstract}
In this note, an explicit trigonometrically-fitted (RK) method is developed to determine the approximate solution of the first-order IVPs with oscillatory solution. The proposed method solves first order ODEs by first converting the second order ODEs to an equivalent first order; which is based on the RK method of order four. The numerical experiment performed shows the efficacy of our newly developed method.
\end{abstract}

Keyword: Trigonometrically-fitted; First order ODEs 